\title{
Patient outcomes in Anteromedial osteoarthritis patients over 80 years old undergoing Oxford Unicompartmental knee Arthroplasty in China
}

\author{
Jingbo Cheng®, Mingli Feng ${ }^{*}$, Guanglei Cao, Zheng Li, Shuai An and Shibao Lu
}

\begin{abstract}
Background: The use of Oxford Unicompartmental Knee Arthroplasty (UKA) has increased rapidly in both Western and Asian populations, with excellent functional outcomes and high patient satisfaction. While previous evidence regarding clinical outcomes and survival rates after Oxford UKA was based on studies in Western populations, the results may be different in Asian patients. The relevance of age for postoperative function after Oxford UKA also remains unclear. Hence, the aim of our study was to clarify the effectiveness and safety of Oxford UKA in Asian patients aged over 80 years.
\end{abstract}

Methods: A retrospective review was performed and included 195 patients (209 knees) who underwent an Oxford UKA between June 2015 and July 2018. We divided the patients into three groups by age: Group 1, 60-69 years; Group 2, 70-79 years; and Group 3, over 80 years. We used the Hospital for Special Surgery (HSS) score and Western Ontario and McMaster (WOMAC) Universities Osteoarthritis Index score to evaluate the general condition of the patients' knees before surgery and at last follow-up. We also recorded perioperative and short-term complications. Result: Group 1 consisted of 60 patients (60 knees); Group 2, 70 patients (79 knees); and Group 3, 65 patients (70 knees). The mean follow-up was $21.34 \pm 12.04,22.08 \pm 11.38$, and $21.76 \pm 10.20$ months in groups 1, 2, and 3, respectively. At last follow-up, the patients in Group 3 showed lower function scores compared to groups 1 and 2 $(P<0.05)$, but the HSS scores and the WOMAC scores were significantly improved in all three groups. In terms of perioperative and other complications, the three age groups did not differ significantly.

Conclusion: Oxford UKA is an effective and safe treatment for osteoarthritis, even in elderly patients in China. Elderly patients have lower knee function scores than younger patients. However, the knee joint pain of the elderly patients was relieved and function improved compared to the preoperative condition.

Keywords: Oxford Unicompartmental knee Arthroplasty, 80 years old, Perioperative complication, Restoration of function, Safety

\footnotetext{
* Correspondence: fengmingli6666@163.com

Department of Orthopaedics, Xuanwu Hospital, Capital Medical University,

Changchun Ave 45, Xicheng District, Beijing 100053, China
}

C The Author(s). 2020 Open Access This article is licensed under a Creative Commons Attribution 4.0 International License, which permits use, sharing, adaptation, distribution and reproduction in any medium or format, as long as you give appropriate credit to the original author(s) and the source, provide a link to the Creative Commons licence, and indicate if changes were made. The images or other third party material in this article are included in the article's Creative Commons licence, unless indicated otherwise in a credit line to the material. If material is not included in the article's Creative Commons licence and your intended use is not permitted by statutory regulation or exceeds the permitted use, you will need to obtain permission directly from the copyright holder. To view a copy of this licence, visit http://creativecommons.org/licenses/by/4.0/ The Creative Commons Public Domain Dedication waiver (http://creativecommons.org/publicdomain/zero/1.0/) applies to the data made available in this article, unless otherwise stated in a credit line to the data. 


\section{Background}

Oxford unicompartmental knee arthroplasty (UKA) is one of most effective surgical procedures for the treatment of isolated medial compartment osteoarthritis. Compared to total knee arthroplasty (TKA), UKA (including both fixed-bearing UKA and mobile-bearing UKA) can provide better physiological function, quicker recovery, shorter hospital stay, and fewer perioperative complications, especially for early arthritis $[1,2]$. Recently, the use of Oxford UKA has increased rapidly around the world, and the effectiveness and safety of a minimally invasive surgical approach for Oxford UKA has demonstrated good-to-excellent long-term follow-up in Western populations $[3,4]$. Today, with the improvement in medical standards, the phenomena surrounding aging is becoming increasingly obvious worldwide. Many elderly patients cannot undergo surgery because of its high risk. Due to the potential reduction of morbidity and mortality compared to TKA, UKA may represent an interesting solution for elderly patients when the disease is limited to medial femorotibial disease.

Asian populations have different lifestyles, such as squatting and sitting on the floor, from those of Western populations. Therefore, Asian populations need more range of motion (as they often flex the knee to more than 120 degrees) to perform daily activities $[5,6]$. A previous study also reports a substantially higher bearing dislocation rate in Asian populations [7], which may relate to the lifestyle-related high degrees of knee flexion. While previous evidence regarding clinical outcomes and survival rates after Oxford UKA were based on studies in Western populations, results may be different in Asian patients. Furthermore, previous studies did not compare different age groups. Thus, the relevance of age in postoperative function after Oxford UKA remains unclear. Hence, the aim of our study was to clarify the effectiveness and safety of Oxford UKA in Asian patients aged over 80 years.

\section{Methods}

\section{Participants}

We retrospectively reviewed 195 knee osteoarthritis patients who underwent medial Oxford UKA (Oxford Unicompartmental Phase 3, Biomet, Warsaw, IN, USA) between June 2015 and July 2018. The study protocol was reviewed and approved by the hospital institutional review board. All patients provided informed consent for participation in the study. All patients were diagnosed with anteromedial osteoarthritis (AMOA) of the knee based on history, physical examination, and radiographs. Before surgery, all patients had standard X-ray views taken: anteroposterior (AP) and lateral radiographs; full-length standing, weight-bearing AP, and lateral radiographs; and patella tangential view radiographs. Magnetic resonance imaging (MRI) was also done to evaluate the ligament, meniscus, and lateral compartment.

The indication criteria for UKA were: older than 55 years; a correctable varus deformity (varus less than 15 degrees); flexion deformity less than 15 degrees; intact ligaments, especially the anterior cruciate ligament (ACL) and medial collateral ligament (MCL) which should be functionally normal; an intact lateral compartment; proper range of motion (at least 0-100 degrees); and no inflammatory disease. We observed the stage of deterioration of the ACL intraoperatively. If the ACL deficiency was less than grade 2 we performed an Oxford UKA (ACL grades: 1. Normal; 2. Loss of synovial covering, usually starting distally; 3 . Longitudinal splits in the substance of the exposed ligament; 4. Friable and fragmented with stretching and loss of strength of the collagen bundles; 5. Absent or ruptured).

Exclusion criteria: The lateral side of the patellofemoral joint exhibited bone loss with eburnation and longitudinal grooving; absent or severely damaged ACL (or PCL or MCL).

\section{Surgical technique and perioperative management}

The same group of surgeons performed all the operations. The patient was placed in the supine position and a tourniquet was applied to the proximal thigh on the operative side. All patients underwent the standard Oxford UKA surgical procedure (minimally invasive Oxford UKA, using the Oxford Microplasty instrumentation). All patients received an analgesic intra-articular cocktail mixture injection containing ropivacaine, parecoxib sodium, oxycodone, epinephrine, and tranexamic acid. Patients received an intravenous infusion of drugs to control pain for 3 days after surgery. All patients routinely received a drainage tube; removed on the first day after surgery. All patients received anticoagulant therapy from 1 day after surgery until 2 weeks after surgery.

\section{Outcome measures}

We used the Hospital for Special Surgery (HSS) Knee score and Western Ontario and McMaster (WOMAC) Universities Osteoarthritis Index to evaluate the general condition of the patients' knees. Preoperative and postoperative range of motion (ROM), HSS, and WOMAC score were used to assess patient function at final follow-up. Postoperative radiographs in three groups, collected at the latest follow-up, were reviewed by the authors to identify any signs of radiolucency, loosening, and progression of arthritis in the lateral and patellofemoral compartments.

Depending on the age of the patients, we divided all patients into three groups (ages 60-69; ages 70-79; age over 80). Patient records were reviewed and the following data collected: American Society of Anesthesiologists 
Table 1 Patient Demographic Characteristics

\begin{tabular}{llll}
\hline Variable & Group 1 & Group 2 & Group 3 \\
\hline Knees & 60 & 79 & 70 \\
Age $(y)$ & $64.93 \pm 3.28$ & $75.17 \pm 4.12$ & $82.41 \pm 2.40$ \\
Sex (male: female) & $26: 34$ & $30: 49$ & $34: 36$ \\
Follow-up $(\mathrm{m})$ & $21.34 \pm 12.04$ & $22.08 \pm 11.38$ & $21.76 \pm 10.20$ \\
\hline
\end{tabular}

(ASA) score, body mass index (BMI), type of anesthesia, length of stay, hemoglobin values (before surgery and three days after surgery), change of hematocrit (before surgery, first and third day after surgery), tourniquet time, and previous basic disease (e.g., hypertension, diabetes, coronary heart disease). We used the Mercurialis method to calculate the volume of blood loss [8]. We used the visual analogue scale (VAS) score to evaluate pain degree eight hours (h), $16 \mathrm{~h}$, and $24 \mathrm{~h}$ after surgery. We also recorded preoperative and postoperative ROM, HSS, and WOMAC scores as well as perioperative and short-term complications.

\section{Statistical analysis}

The statistical software used for all analyses was SPSS 22.0 (SPSS Inc., Chicago, IL, USA). Continuous variables are reported as means \pm standard deviation (with range). Discrete variables are reported as number (percent). Chi-squared test or Fisher's exact probability method was used to compare binary variables (demographic data and complication rates). We used analysis of variance (ANOVA) for comparison of clinical scores (HSS score, WOMAC score) between the three groups (significance set at $P<0.05)$.

The minimum clinically important differences (MCID) of the WOMAC score was 11 for pain, 9 for function, 8 for stiffness, and 10 for the total WOMAC score [9]. The MCID of HSS score was 6 according to previous study [10]. Our sample size was sufficient to detect potentially relevant differences regarding this clinical parameter.

One-way ANOVA was used for the comparison of the means between the three groups. Homogeneity of variance is needed before conducting ANOVA. First, we calculated a global/overall model. Only in case of significance, Bonferroni adjusted pairwise comparisons are needed to detect which groups differ significantly. The binary variables between the multiple groups is expressed in percentage (P) and was compared using Fisher's exact probability method or chi-square test.

\section{Results}

One hundred ninety five patients enrolled in our study. Among the patients, 60 (60 knees) were 60-69 years old (Group 1); 70 patients (79 knees), 70-79 years old (Group 2); and 65 patients (70 knees), older than 80 years (Group 3). The mean follow-up was $21.34 \pm 12.04$, $22.08 \pm 11.38$, and $21.76 \pm 10.20$ months in groups 1,2 ,

Table 2 Comparison of Patient Characteristics, and Preoperative Variables Between different groups

\begin{tabular}{|c|c|c|c|c|c|c|}
\hline Variable & Group 1 & Group 2 & Group 3 & $\mathrm{~F}$ value/X2 & $\eta 2$ & $P$ Value \\
\hline ASA score & $1.5 \pm 0.5$ & $1.5 \pm 0.5$ & $1.5 \pm 0.5$ & 0.617 & 0.012 & 0.723 \\
\hline $1-2$ & $51(85.0 \%)$ & $64(81.0 \%)$ & $51(72.9 \%)$ & & & \\
\hline $3-4$ & $9(15.0 \%)$ & 15 (19.0\%) & $19(27.1 \%)$ & & & \\
\hline BMl & $27.35 \pm 3.64$ & $27.19 \pm 3.82$ & $26.84 \pm 3.61$ & 0.125 & 0.007 & 0.932 \\
\hline Normal $(<25)$ & $21(35.0 \%)$ & $28(35.5 \%)$ & $26(37.1 \%)$ & & & \\
\hline Overweight (25-30) & $30(50.0 \%)$ & 31 (39.2\%) & $30(42.9 \%)$ & & & \\
\hline Obese (> 30) & $9(15.0 \%)$ & $20(25.3 \%)$ & $14(20.0 \%)$ & & & \\
\hline \multicolumn{7}{|l|}{ Anesthesia } \\
\hline General & $1(1.7 \%)$ & $2(2.5 \%)$ & $10(14.3 \%)$ & & & \\
\hline Spine & 59 (98.3\%\%) & 77 (97.5\%) & $60(85.7 \%)$ & & & \\
\hline Hypertension & $35(58.3 \%)$ & $48(60.7 \%)$ & $57(81.4 \%)^{*}$ & 8.192 & / & 0.036 \\
\hline Diabetes & $18(30.0 \%)$ & $24(30.4 \%)$ & 19 (27.1\%) & 0.663 & / & 0.792 \\
\hline Coronary heart disease & $14(23.3 \%)$ & 19 (24.1\%) & $20(28.5 \%)$ & 0.386 & / & 0.697 \\
\hline Digestive diseases & $5(8.3 \%)$ & $7(8.8 \%)$ & $7(10.0 \%)$ & 0.286 & / & 0.711 \\
\hline Nervous system disease & $6(10.0 \%)$ & $9(11.4 \%)$ & $12(17.1 \%)$ & 2.861 & / & 0.323 \\
\hline Immune system disease & $1(1.7 \%)$ & $2(2.5 \%)$ & 0 & 2.102 & / & 0.283 \\
\hline Respiratory diseases & $2(3.3 \%)$ & $3(3.8 \%)$ & $7(10.0 \%)$ & 3.287 & / & 0.113 \\
\hline Peripheral vascular disease & $1(1.7 \%)$ & $2(2.5 \%)$ & $6(8.5 \%)$ & 1.983 & / & 0.204 \\
\hline Coagulation abnormalities & $8(13.3 \%)$ & $12(15.2 \%)$ & $11(15.7 \%)$ & 0.582 & / & 0.841 \\
\hline
\end{tabular}

"*" Represent compared with the Group 1and 2, the difference in Group 3 is statistically different 
and 3, respectively. Table 1 describes the general conditions of all the patients.

The patients' characteristics and perioperative variables between different groups are shown in Table 2. In Group 3, the number of patients with hypertension was statistically higher than that of groups 1 and $2(p<0.05)$. There were no significant differences in ASA scores, BMI, and other basic diseases between the three groups. There were no significant differences in preoperative ROM.

The postoperative variables and perioperative complications between the different groups are shown in Table 3. There were no significant differences in hospital stay, tourniquet time, changes of hemoglobin, blood loss volume, or postoperative ROM between the three groups. Regarding perioperative complications, the risk of superficial wound infection and wound swelling in elderly patients was slightly higher than that in patients under 80 years old $(p<0.05)$. A total of 13 patients suffered superficial infection after surgery. They received vacuum sealing drainage (VSD) and recovered. There were no significant differences in other perioperative complications.

There were also no statistical differences in preoperative and postoperative HSS or WOMAC scores (Table 4). Although there was no statistical difference between the three groups, the knee scores of older patients were still lower than those of the relatively younger patients. Compared to before surgery, significant improvements were found in HSS and WOMAC scores among the three groups. Compared to groups 1 and 2, Group $3(p<0.05)$ showed a lower mean function score in HSS and WOMAC scores, but this was improved compared to the preoperative condition. The MCID of the WOMAC score was 10 and the MCID of HSS score was $6[9,10]$. These differences may be of little importance. There were no significant differences in postoperative VAS scores between the three groups.

Table 5 describes the surgery-related complications after Oxford UKA. One patient in Group 1 developed loosening of the tibial component 2 years after surgery, but did not have any complaint of discomfort. We are continuing clinical follow-up of this patient. Group 1 had 5 patients found to have radiolucent lines at final follow-up; Group 2, 5 patients; and Group 3, 9 patients. One patient in Group 1 suffered bearing dislocation four months after surgery and subsequently received a TKA. One patient in Group 3 accidentally fell two months after surgery and was diagnosed with a periprosthetic fracture. This patient later underwent open reduction of the tibial fracture with internal fixation (LCP Medial Proximal Tibial Plate).

\section{Discussion}

There were no major systemic complications following 209 consecutive Oxford UKAs (195 patients). This study revealed no significant differences in the perioperative complication rates in patients between the different

Table 3 Comparison of postoperative variables and Perioperative complications between different groups

\begin{tabular}{|c|c|c|c|c|c|c|}
\hline Variable & Group 1 & Group 2 & Group 3 & F value/ $x^{2}$ & $\eta 2$ & $P$ Value \\
\hline Hospital stay (d) & $10.54 \pm 3.37$ & $11.23 \pm 2.82$ & $12.64 \pm 2.68$ & 1.276 & 0.009 & 0.387 \\
\hline Tourniquet time (min) & $72.50 \pm 6.57$ & $75.16 \pm 9.53$ & $76.23 \pm 11.18$ & 1.785 & 0.012 & 0.204 \\
\hline Changes of hemoglobin ( $\mathrm{g} / \mathrm{L})$ & $14.11 \pm 7.48$ & $15.29 \pm 9.39$ & $13.05 \pm 8.69$ & 0.967 & 0.026 & 0.458 \\
\hline Blood loss volume (ml) & $178.17 \pm 74.73$ & $179.80 \pm 84.22$ & $175.50 \pm 80.39$ & 0.641 & 0.034 & 0.783 \\
\hline Preoperative ROM & $111.91 \pm 11.57$ & $108.83 \pm 10.84$ & $103.86 \pm 10.68$ & 0.884 & 0.075 & 0.539 \\
\hline Postoperative ROM & $120.23 \pm 7.99$ & $115.38 \pm 8.27$ & $112.95 \pm 7.01$ & 0.923 & 0.115 & 0.413 \\
\hline \multicolumn{7}{|l|}{ Perioperative complications } \\
\hline Myocardial infarction & 0 & 0 & 0 & & & \\
\hline Congestive heart failure & $1(1.6 \%)$ & 0 & 0 & 0.598 & / & 0.567 \\
\hline Cerebrovascular accident & 0 & 0 & 0 & & & \\
\hline Lung infection & $1(1.6 \%)$ & $1(1.2 \%)$ & $2(2.9 \%)$ & 0.682 & / & 0.542 \\
\hline Pulmonary embolism & 0 & 0 & 0 & & & \\
\hline Urinary system infection & 0 & 0 & 0 & & & \\
\hline Abnormal renal and liver function & $2(3.3 \%)$ & $4(5.1 \%)$ & 0 & 3.117 & / & 0.195 \\
\hline Deep vein thrombosis & $3(5.0 \%)$ & $5(6.3 \%)$ & $5(7.1 \%)$ & 0.265 & / & 0.838 \\
\hline Calf muscular vein thrombosis & $9(15.0 \%)$ & $14(17.7 \%)$ & $13(18.5 \%)$ & 0.386 & / & 0.643 \\
\hline Hypoproteinemia & $8(13.3 \%)$ & $9(11.4 \%)$ & $11(15.7 \%)$ & 1.487 & / & 0.455 \\
\hline Superficial infection & $1(1.6 \%)$ & $1(1.2 \%)$ & $11(15.7 \%)^{*}$ & 11.248 & / & 0.015 \\
\hline Deep infection & 0 & 0 & 0 & & & \\
\hline Swelling of the wound & $4(6.6 \%)$ & $5(6.3 \%)$ & $12(17.1 \%)^{*}$ & 10.749 & / & 0.023 \\
\hline
\end{tabular}

"**" Represent compared with the Group 1and 2, the difference in Group 3 is statistically different 
Table 4 Comparison of preoperative and Postoperative Knee score between different groups

\begin{tabular}{|c|c|c|c|c|c|c|}
\hline & Group 1 & Group 2 & Group 3 & F Value & $\eta 2$ & $P$ Value \\
\hline VAS score (8 h after surgery) & $1.08 \pm 0.83$ & $1.29 \pm 0.56$ & $1.25 \pm 0.57$ & 0.912 & 0.019 & 0.608 \\
\hline VAS score ( $16 \mathrm{~h}$ after surgery) & $1.65 \pm 0.76$ & $1.83 \pm 0.79$ & $1.80 \pm 0.72$ & 0.823 & 0.009 & 0.590 \\
\hline VAS score ( $24 \mathrm{~h}$ after surgery) & $1.43 \pm 0.48$ & $1.73 \pm 0.42$ & $1.54 \pm 0.56$ & 1.023 & 0.021 & 0.667 \\
\hline Preoperative HSS & $61.74 \pm 6.96$ & $58.13 \pm 7.59$ & $55.68 \pm 7.53$ & 1.468 & 0.092 & 0.147 \\
\hline Function score & $13.96 \pm 3.08$ & $12.59 \pm 2.36$ & $11.09 \pm 2.60$ & 1.997 & 0.091 & 0.160 \\
\hline Pain score & $13.20 \pm 6.27$ & $13.00 \pm 5.28$ & $12.27 \pm 4.30$ & 0.921 & 0.059 & 0.513 \\
\hline Preoperative WOMAC & $44.30 \pm 11.26$ & $46.85 \pm 14.90$ & $48.91 \pm 13.10$ & 1.342 & 0.134 & 0.208 \\
\hline Function score & $32.28 \pm 10.27$ & $33.28 \pm 12.27$ & $36.68 \pm 11.58$ & 2.864 & 0.099 & 0.140 \\
\hline Pain score & $11.60 \pm 3.86$ & $10.50 \pm 4.06$ & $10.88 \pm 4.63$ & 0.864 & 0.091 & 0.684 \\
\hline Stiffness & $3.01 \pm 1.98$ & $2.98 .50 \pm 3.06$ & $3.21 \pm 2.11$ & 0.819 & 0.089 & 0.798 \\
\hline Postoperative HSS & $86.61 \pm 6.38$ & $85.23 \pm 6.98$ & $83.09 \pm 6.04$ & 2.128 & 0.206 & 0.129 \\
\hline Function score & $19.34 \pm 2.56$ & $18.34 \pm 2.59$ & $16.64 \pm 1.56^{*}$ & 15.736 & 0.125 & $<0.001$ \\
\hline Pain score & $5.96 \pm 2.08$ & $5.39 \pm 3.14$ & $5.76 \pm 3.08$ & 0.866 & 0.085 & 0.785 \\
\hline Postoperative WOMAC & $24.16 \pm 10.53$ & $25.56 \pm 10.53$ & $28.00 \pm 9.50$ & 3.437 & 0.233 & 0.086 \\
\hline Function score & $14.28 \pm 5.53$ & $13.89 \pm 4.69$ & $18.23 \pm 5.81^{*}$ & 10.289 & 0.134 & $<0.001$ \\
\hline Pain score & $5.29 \pm 3.19$ & $5.46 \pm 4.42$ & $5.76 \pm 3.08$ & 0.854 & 0.104 & 0.781 \\
\hline Stiffness & $2.26 \pm 1.36$ & $1.98 \pm 1.43$ & $2.24 \pm 1.14$ & 1.876 & 0.201 & 0.620 \\
\hline Change of HSS & $24.87 \pm 6.67$ & $27.10 \pm 6.83$ & $27.40 \pm 6.02$ & 2.145 & 0.001 & 0.108 \\
\hline Change of HSS function score & $5.68 \pm 3.21$ & $6.21 \pm 3.02$ & $5.85 \pm 4.15$ & 0.987 & 0.018 & 0.698 \\
\hline Change of WOMAC & $20.93 \pm 4.82$ & $21.90 \pm 5.39$ & $20.91 \pm 4.68$ & 0.853 & 0.021 & 0.728 \\
\hline Change of WOMAC function score & $19.18 \pm 8.01$ & $20.08 \pm 6.99$ & $18.00 \pm 7.10$ & 1.256 & 0.013 & 0.657 \\
\hline
\end{tabular}

"**" Represent compared with the Group 1and 2, the difference in Group 3 is statistically different

groups. All patients obtained satisfactory clinical outcomes, but compared to patients over 80 years old, patients between the ages of 60-79 had a higher function score in both HSS and WOMAC scores.

\section{Clinical outcome of UKA}

Iacono et al. [11] evaluated results obtained in patients older than 75 years treated with UKA. All clinical scores improved significantly at follow-up, and the outcome was considered good or excellent in $92.6 \%$ of the patients, but the prosthesis used was different from ours.

Concerning the clinical outcomes in very old patients who underwent Oxford UKA, a recent study from the Oxford

Table 5 The surgery-related complications after Oxford UKA

\begin{tabular}{llll}
\hline Complication & Group 1 & Group 2 & Group 3 \\
\hline Implant loosening & 1 & 0 & 0 \\
Radiolucency & 5 & 5 & 9 \\
Dislocation & 1 & 0 & 0 \\
Periprosthetic fracture & 0 & 0 & 1 \\
Periprosthetic joint infection & 0 & 0 & 0 \\
Progression of arthritis & 0 & 0 & 0 \\
Persistent unexplained pain & 4 & 2 & 3 \\
\hline
\end{tabular}

center analyzed 1000 Oxford UKAs and found that at 10year follow-up, patients younger than 60 at the time of the operation had significantly better American Knee Society Score Function (AKSS-F) score, Oxford Knee Score (OKS), and Tegner Activity Score than patients older than 60, but no difference in functional outcomes was seen between the groups [12]. A meta-analysis reported that the functional outcome of UKA in the elderly is good, with low rates of perioperative morbidity and mortality [13]. Inale et al. [14] reviewed the short-term results of mobile-bearing medial UKA in elderly patients and compared the results with younger patients. The differences between the knee scores from the elderly patients and from the younger patients were not statistically significant. Revision rate and survival of the implant were not different among the groups.

In our study, there was a clear improvement in HSS and WOMAC scores in both groups after surgery. WOMAC scores evaluate efficacy through three aspects: function, pain, and stiffness. HSS scores evaluate efficacy through two aspects: function and pain. There was no statistical difference between the three groups in the total HSS score and WOMAC score. However, Group 3 had lower scores in the functional dimension in HSS and WOMAC scores, and there was no statistical difference in the pain aspects in HSS and WOMAC scores. 
According to previous study $[9,10]$, the MCID of the WOMAC score and HSS was 10 and 6.This is considered small and still within the MCID of the function outcome measurement. Thus, even though the difference is statistically significant, it might not be clinically important. The lack of exercise and the decline of activity by patients older than 80 might also have led to this finding.

In Asian populations, body size, BMI, lifestyle, and knee morphology of Asian populations differ from those in Western countries. A proportion of patients, whose knees flex more than 120 degrees, are required to perform daily activities that include squatting and sitting on the floor, which may lead to different clinical outcomes from Western populations. Lim et al. reported that Oxford UKA can yield satisfactory clinical and functional results and has a 10-year survival rate of $94 \%$ in Korean patients [7]. Yoshida et al. report similarly good medium-term results with a 10-year survival rate of $95 \%$ in Japanese patients [15]. In our study, the clinical outcome of Chinese patients is similar to Western patients' clinical outcome, and the ROM also changes significantly in both groups before and after surgery.

\section{Surgery-related complications}

One systematic review assessed over 8000 Oxford UKA patients and found the 10-year survival to be $93 \%, 15$ year survival to be $89 \%$, and a medical complication incidence of $0.8 \%$. Very good outcomes were achieved by both designing and non-designing surgeons [16]. The literature shows that the main reasons that led to failures of Oxford UKA were bearing dislocation, aseptic loosening, lateral compartment arthritis progression, and persistent unexplained pain [16-20]. Of the 209 Oxford UKAs in our study, 19 (9.1\%) patients were found to have radiolucent lines (RLL) under the tibial component on radiographs at final follow-up. This is different from the results reported by other studies. Previous literature shows that the incidence of RLL ranged from 62 to $96 \%$, which was not clinically related to inferior functional outcomes [21-24]. The etiology of radiolucency remains unknown. The incidence of RLL in the current study was lower than in the previous literature. Several reasons may lead to this phenomenon, such as the small sample size, short follow-up time, and lack of standard X-rays (we could usually not get standard X-rays for outpatient follow-ups). Goodfellow et al. describe pathological RLL being $>2 \mathrm{~mm}$ thickness, poorly defined, and often related to aseptic loosening. On the contrary, physiological RLL are 1-2 mm thick, and well-defined [25]. The presence of RLL in our patients was not related to their symptoms or indicative or predictive of loosening and, according to the $\mathrm{X}$-rays, we confirmed them to be physiological RLL (Fig. 1). We still need to assess the clinical outcomes through mid- and long-term follow-ups.

One patient suffered a bearing dislocation four months after surgery. Figure 2 shows the imaging before and after surgery. The possible reason for this may be that the abnormal morphology of the patient's femur lead to a deviation
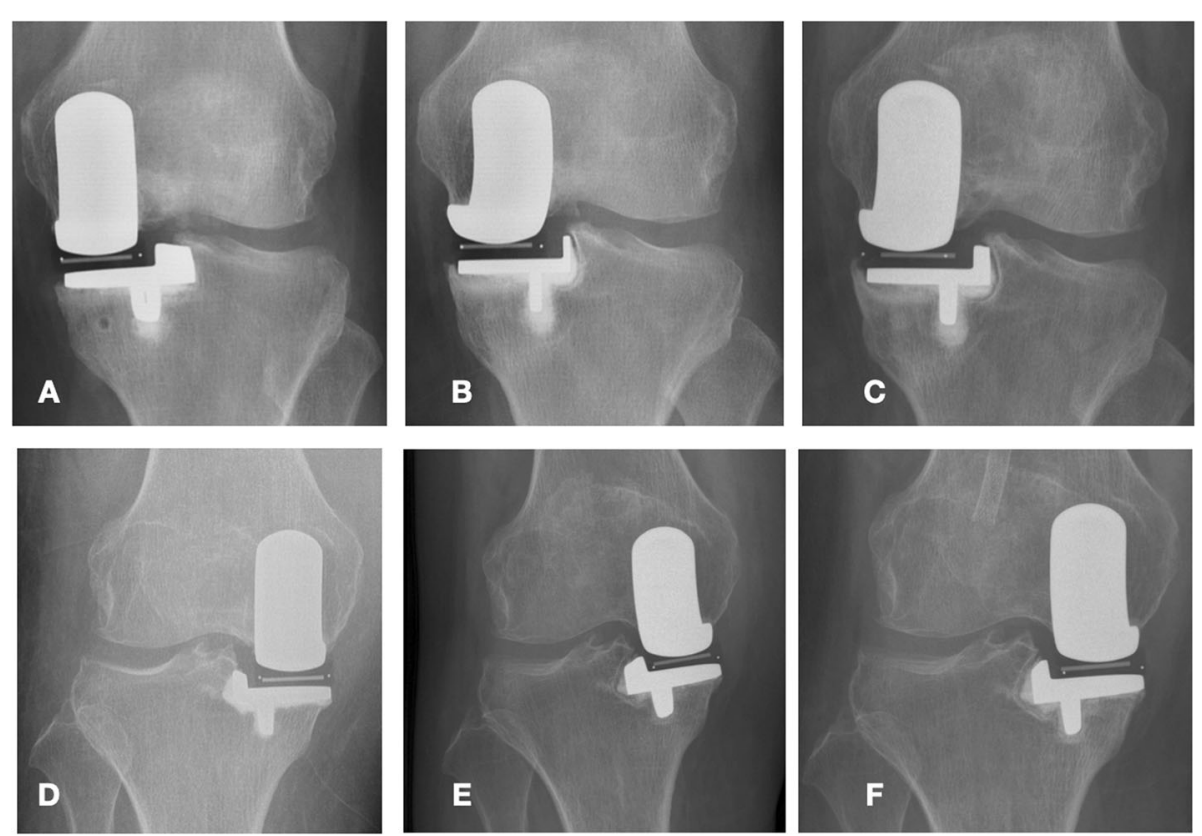

Fig. 1 shows 2 patients developed radiolucent line after surgery ( 6 months after surgery, 1 year after surgery), both the 2 patients did not have pain or other symptoms. $\mathbf{a}, \mathbf{b}$ and $\mathbf{c}$ are the X-rays of the patient 3 months after surgery, 1 year after surgery and 2 years after surgery. $\mathbf{d}, \mathbf{e}$ and $\mathbf{f}$ are the $\mathrm{X}$-rays of another patient 2 weeks after surgery, 6 months after surgery and 1 year after surgery 

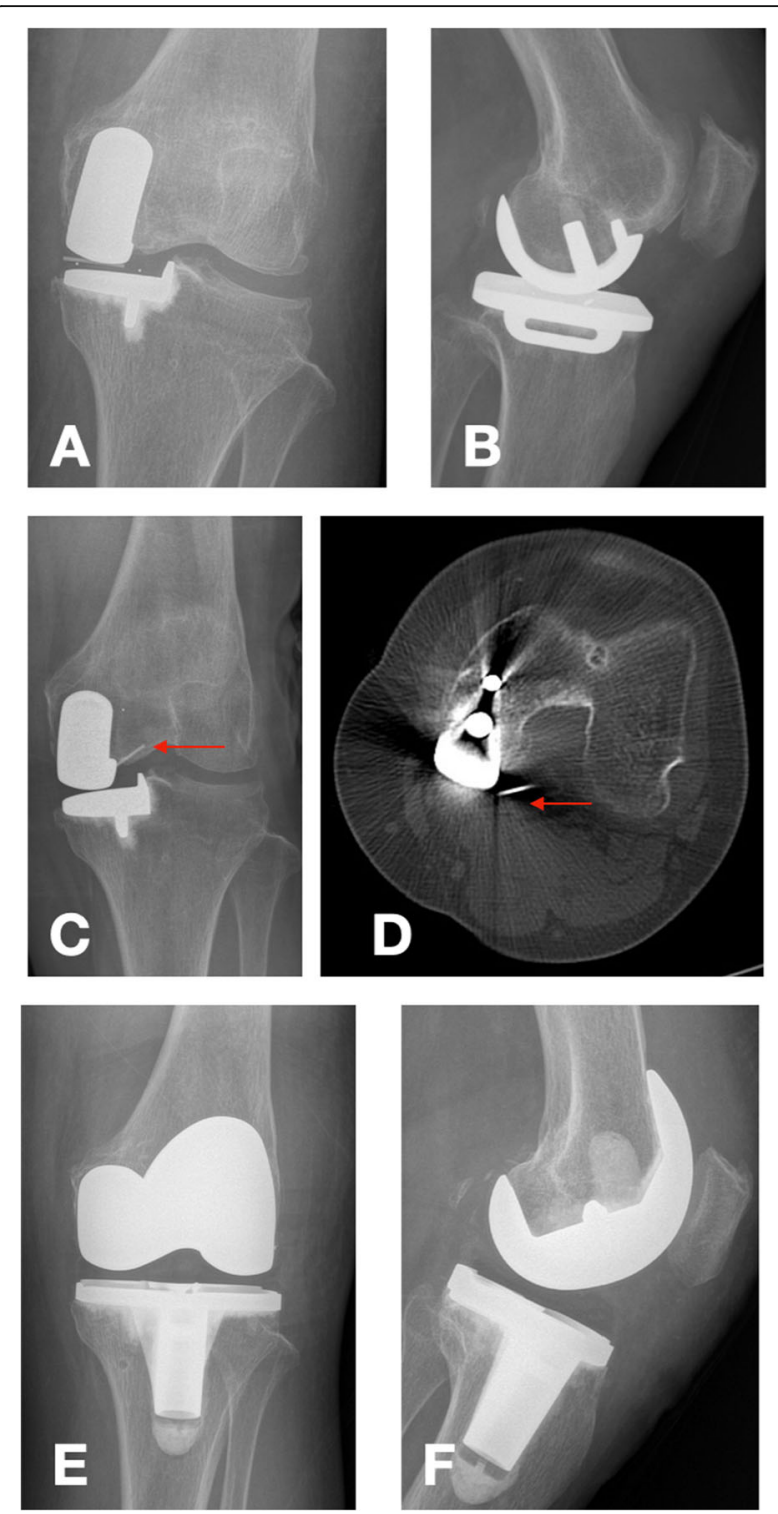

Fig. 2 shows a 64-year-old woman suffered moderate pain 4 months after surgery, and the patients received X-ray and CT scan for examination. From the data of imaging we found bearing dislocation. Later the patient performed TKA and got satisfactory clinical outcome after TKA. $\mathbf{a}$ and $\mathbf{b}$ are the X-rays of the patient 2 weeks after surgery. $\mathbf{c}$ is the $\mathrm{X}$-ray of the patient 4 months after surgery. $\mathbf{d}$ is the CT scan of the patient 4 months after surgery. $\mathbf{e}$ and $\mathbf{f}$ are the $\mathrm{X}$-ray of the patient 1 year after TKA surgery

in the intramedullary rod positioning and the femoral prosthesis was placed medially. The poor prosthesis position caused rotation of the bearing during knee flexion, resulting in dislocation of the bearing. Bearing dislocation is a major complication of Oxford UKA, as previous literature has reported, and the rate of bearing dislocation is higher in Asian populations than in Western populations [5, 26, 27]. It can occur in the presence of an unbalanced flexionextension gap, impingement of the bearing with adjacent bone or the tibial/femoral component, instability of the medial compartment due to MCL injury, or secondary to femoral/tibial component loosening [5].

One patient (81 years old) developed a periprosthetic fracture 2 months after surgery due to a fall, and we performed an open reduction of the tibial fracture with internal fixation. Figure 3 shows the X-ray before and after surgery. The literature shows that the rates of fracture in knee arthroplasties are reported to be from $0.2-2.5 \%$ in clinical studies and $0.02-0.17 \%$ in worldwide arthroplasty registers [28]. Risk factors associated with unicompartmental component periprosthetic fracture include malalignment with increased local stresses due to malpositioning, progressive osteoarthritis, and cruciate ligament deficiency. Patients with a BMI greater than 30 are also at greater risk [29].

\section{Perioperative complications}

The major perioperative complications in our study were calf muscular vein thrombosis (CMVT) and superficial infection. There were no deaths during the perioperative period, pulmonary embolisms, or symptomatic deep vein thrombosis (DVT).

Chan et al. compared one-stage and two-stage bilateral unicompartmental knee replacements during the first 30 days postoperatively and found that the rates of proximal DVT, pulmonary embolus, and death secondary to pulmonary embolus to be $0.9,1.9$, and $0.3 \%$, respectively [30]. If the patient was diagnosed with a DVT or CMVT, they should receive low molecular weight heparin (nadroparin $0.4 \mathrm{~mL}$, twice per day) for 2 weeks. Two weeks after surgery, patients were treated with Rivaroxaban for anticoagulant therapy. The deep vein ultrasound of the lower extremity should then be checked and the drug stopped if the thrombus disappears or dissolves. Other patients received low molecular weight heparin (nadroparin $0.4 \mathrm{~mL}$ once per day) after surgery. In total, 13 patients developed superficial infection after surgery (11 patients older than 80). Patient-related risk factors included previous revision arthroplasty, previous infection associated with a prosthetic joint at the same site, tobacco use, obesity, rheumatoid arthritis, a neoplasm, immunosuppression, and diabetes mellitus [31]. Postoperative risk factors included incision healing complications (e.g., superficial infection, hematoma, delayed healing, incision necrosis, and dehiscence), atrial fibrillation, myocardial infarction, urinary tract infection, and prolonged hospital stay [31, 32].

We conclude that Oxford UKA is a safe procedure with a low rate of perioperative complications, similar to previous studies [33]. Previous studies also showed that increased patient age and history of cardiovascular disease were identified as risk factors for perioperative death in TKA [34]. However, in our study, patients older 

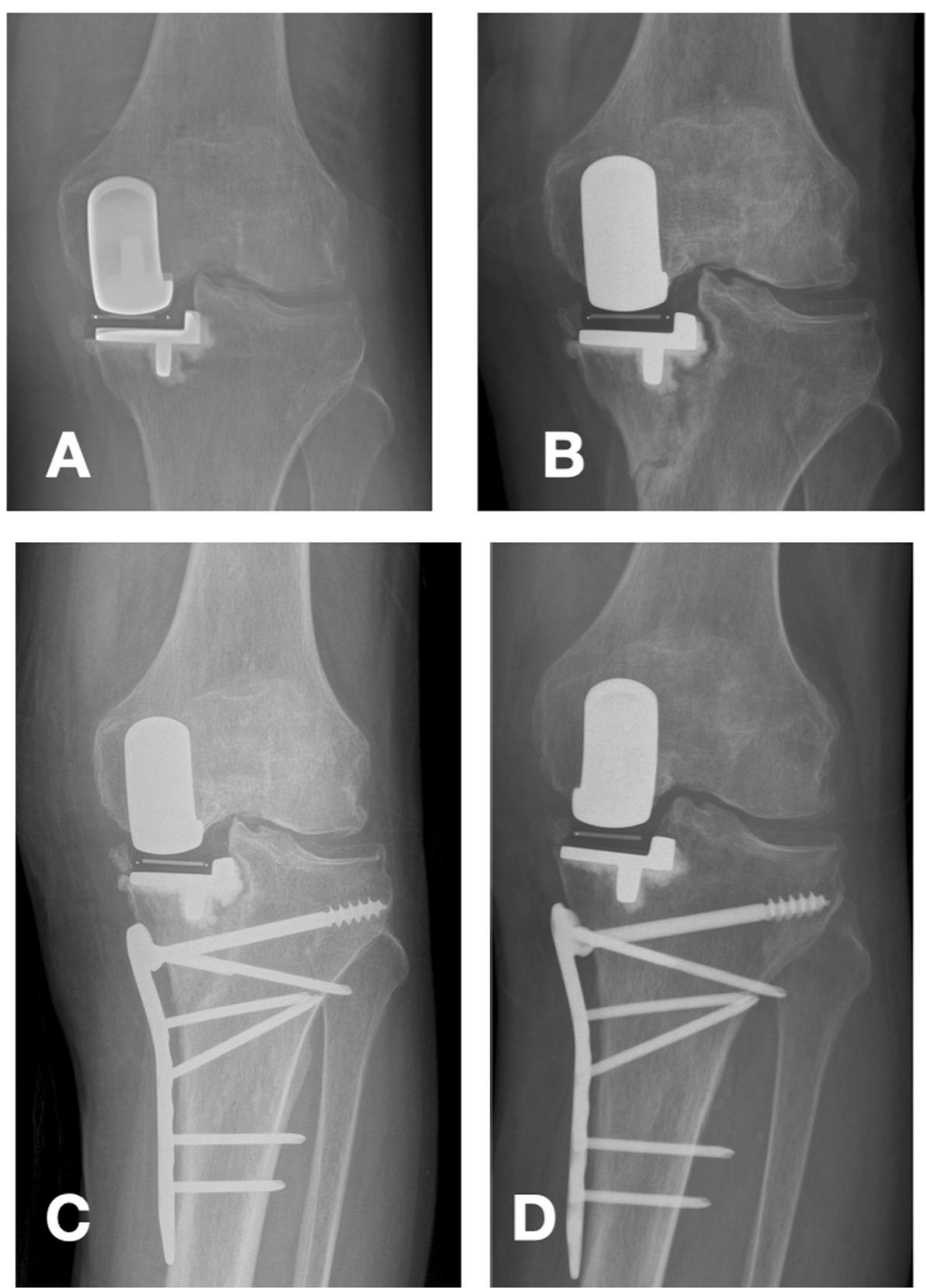

Fig. 3 shows an 81-year-old woman fell down 2 months after surgery and later performed open reduction of tibial fracture with internal fixation. $\mathbf{a}$ is the $\mathbf{X}$-rays of the patient 2 weeks after surgery. $\mathbf{b}$ is the X-rays of the patient 2 months after surgery. $\mathbf{c}$ is the $X$-ray of the patient 3 days after plate fixation. $\mathbf{d}$ is the X-ray of the patient 8 months after plate fixation

than 80 who underwent Oxford UKA also showed good clinical outcomes with a low rate of perioperative and other complications.

\section{Limitations}

There are several limitations in our study. The study sample was relatively small, and the follow-up relatively short. Further research, large samples, and long-term follow-up are required to evaluate function. The mean follow-up time of the study group in the present study was 21.76 months, which is comparatively long-term if the entry age of 80 years is considered. Moreover, we did not consider the potential influence of sex, and there was no control group in this study.

\section{Conclusion}

Oxford UKA is an effective and safe treatment for osteoarthritis, even in elderly adult patients in China. The knee joint pain symptoms of elderly patients are relieved, and the function improved but was still poor compared with younger patients. The rate of perioperative and other complications in the elderly patients was almost the same as in younger patients. Our study shows the safe use of Oxford UKA in octogenarians in China.

\section{Abbreviations}

ACL: Anterior cruciate ligament; AKSS-F: American Knee Society Score Function score; AMOA: Anteromedial osteoarthritis; ANOVA: Analysis of variance; AP: Anteroposterior; ASA: American Society of Anesthesiologists; BMI: Body mass index; CDFI: Color Doppler flow imaging; CMVT: Calf muscular vein thrombosis; DVT: Deep vein thrombosis; HSS: Hospital for Special Surgery knee score; MCID: Minimum clinically important difference; 
MCL: Medial collateral ligament; MRI: Magnetic resonance imaging; OKS: Oxford Knee Score; RLL: Radiolucent lines; ROM: Range of motion; TKA: Total knee arthroplasty; UKA: Unicompartmental knee arthroplasty; VAS: Visual analogue scale score; VSD: Vacuum sealing drainage; WOMAC: Western Ontario and McMaster (WOMAC) Universities Osteoarthritis Index

\section{Acknowledgments}

We express deep thanks to Dr. Mingli Feng and Guanglei Cao for their technical support.

\section{Authors' contributions}

$J B C, M L F$, and SBL conceived and designed the experiments. MLF, ZL, and GLC performed the surgeries. SA and JBC analyzed the data. JBC prepared the figures. JBC wrote the main manuscript text. All authors reviewed the manuscript. All authors read and approved the final manuscript.

\section{Funding}

No funding was received.

\section{Availability of data and materials}

The datasets analyzed during the current study are available from the corresponding author on reasonable request.

\section{Ethics approval and consent to participate}

The research has been conducted in accordance with the Declaration of Helsinki. All experimental protocols were approved by Xuanwu Hospital Capital Medical University Committee. Written informed consent was obtained from all patients.

\section{Consent for publication}

We obtained permission from the participants to publish their data. All participants gave written consent for publication.

\section{Competing interests}

The authors declare that they have no competing interests.

Received: 19 November 2019 Accepted: 2 July 2020

Published online: 08 July 2020

\section{References}

1. Lyons MCMS, Somerville LE, Naudie DD, McCalden RW. Unicompartmental versus Total knee Arthroplasty database analysis. Clin Orthop Relat Res. 2012:470(1):84-90.

2. Kulshrestha V, Datta B, Kumar S, Mittal G. Outcome of Unicondylar knee Arthroplasty vs Total knee Arthroplasty for early medial compartment arthritis: a randomized study. J Arthroplast. 2017;32(5):1460-9.

3. Lisowski LA ML, van den Bekerom MP, Pilot P, Lisowski AE. Ten- to 15-year results of the Oxford Phase III mobile unicompartmental knee arthroplasty: a prospective study from a non-designer group. Bone Joint J. 2016;98 B(10 supple B):41-47.

4. Pandit HHT, Jenkins C, Mellon SJ, Dodd CA, Murray DW. The clinical outcome of minimally invasive phase 3 Oxford unicompartmental knee arthroplasty: a 15-year follow-up of 1000 UKAs. Bone Joint J. 2015;97-B(11): 1493-500.

5. Kim SJPR, Koo S, Kim JH. Causes of revision following Oxford phase 3 unicompartmental knee arthroplasty. Knee Surg Sports Traumatol Arthrosc 2014;22(8):1895-901.

6. Choy WS, Lee KW, Kim HY, Kim KJ, Chun YS, Yang DS. Mobile bearing medial unicompartmental knee arthroplasty in patients whose lifestyles involve high degrees of knee flexion: a 10-14 year follow-up study. Knee. 2017;24(4):829-36

7. Lim HCBJ, Song SH, Kim SJ. Oxford phase 3 unicompartmental knee replacement in Korean patients. J Bone Joint Surg Br. 2012;94(8):1071-6.

8. Mercuriali FIG. Proposal of an algorithm to help the choice of the best transfusion strategy. Curr Med Res Opin. 1996;13(8):465-78.

9. Nicholas D, Clement MB, Weir D, Holland J, Gerrand C, Deehan DJ. What is the minimum clinically important difference for the WOMAC index after TKA? Clin Orthop Relat Res. 2018;476(10):2005-14.

10. C-ST HRBAR, Chen YJD, Pang H-N, Tay K-JD, Chin P-L, Chia S-L, Lo N-N, Yeo S-J. Age and preoperative knee society score are significant predictors of outcomes among Asians following Total knee Arthroplasty. J Bone Joint Surg Am. 2016:98(9):735-41.

11. lacono F, Raspugli GF, Akkawi I, Bruni D, Filardo G, Budeyri A, et al. Unicompartmental knee arthroplasty in patients over 75 years: a definitive solution? Arch Orthop Trauma Surg. 2016;136(1):117-23.

12. Hamilton TWPH, Jenkins C, Mellon SJ, Dodd CAF, Murray DW. Evidence-based indications for Mobile-bearing Unicompartmental knee Arthroplasty in a consecutive cohort of thousand knees. J Arthroplast. 2017;32(6):1779-85.

13. Howieson AFW. Unicompartmental knee replacement in the elderly: a systematic review. Acta Orthop Belg. 2015;81(4):565-71.

14. Ingale PA, Hadden WA. A review of mobile bearing unicompartmental knee in patients aged 80 years or older and comparison with younger groups. J Arthroplast. 2013;28(2):262-7 e2.

15. Yoshida KTM, Yoshida H, Takei S, Fukuoka S, Nakamura H. Oxford phase 3 unicompartmental knee arthroplasty in Japan--clinical results in greater than one thousand cases over ten years. J Arthroplast. 2013;28(9 Suppl):168-71.

16. Mohammad HR, Strickland L, Hamilton TW, Murray DW. Long-term outcomes of over 8,000 medial Oxford phase 3 Unicompartmental knees-a systematic review. Acta Orthop. 2018;89(1):101-7.

17. Peersman GSB, Vandenlangenbergh T, Cartier P, Fennema P. Fixed- versus mobile-bearing UKA: a systematic review and meta-analysis. Knee Surg Sports Traumatol Arthrosc. 2015:23(11):3296-305.

18. Ko YB, Gujarathi MR, Oh KJ. Outcome of Unicompartmental knee Arthroplasty: a systematic review of comparative studies between fixed and Mobile bearings focusing on complications. Knee Surg Relat Res. 2015;27(3):141-8.

19. Neufeld MEAA, Greidanus NV, Garbuz DS, Masri BA. A comparison of Mobile and fixed-bearing Unicompartmental knee Arthroplasty at a minimum 10year follow-up. J Arthroplast. 2018;33(6):1713-8.

20. van der List JPZH, Pearle AD. Why do medial Unicompartmental knee Arthroplasties fail today? J Arthroplast. 2016;31(5):1016-21.

21. Tibrewal SBGK, Goodfellow JW. The radiolucent line beneath the tibial components of the Oxford meniscal knee. J Bone Joint Surg Br. 1984;66(4):523-8

22. Gulati ACR, Pandit HG, Gray H, Price AJ, Dodd CA, Murray DW. The incidence of physiological radiolucency following Oxford unicompartmental knee replacement and its relationship to outcome. J Bone Joint Surg Br. 2009;91(7):896-902.

23. Kleeblad LJ, van der List JP, Zuiderbaan HA, Pearle AD. Regional femoral and Tibial radiolucency in cemented Unicompartmental knee Arthroplasty and the relationship to functional outcomes. J Arthroplast. 2017:32(11):3345-51.

24. Kalra SST, Berko B, Walton NP. Assessment of radiolucent lines around the Oxford unicompartmental knee replacement: sensitivity and specificity for loosening. J Bone Joint Surg Br. 2011;93(6).

25. Goodfellow JOCJ, Dodd C, Murray D. Unicompartmental arthroplasty with the Oxford knee. Oxford: Oxford University Press; 2006. p. 117-28.

26. Choy WSKK, Lee SK, Yang DS, Kim CM, Park JS. Medial unicompartmental knee arthroplasty in patients with spontaneous osteonecrosis of the knee. Clin Orthop Surg. 2011;3(4):279-84.

27. Kim KTLS, Park HS, Cho KH, Kim KS. A prospective analysis of Oxford phase 3 unicompartmental knee arthroplasty. Orthopedics. 2007;30(5 Suppl):15-8.

28. Gilg MM, Zeller CW, Leitner L, Leithner A, Labek G, Sadoghi P. The incidence of implant fractures after knee arthroplasty. Knee Surg Sports Traumatol Arthrosc. 2016;24(10):3272-9.

29. Manzotti ACC, Pullen C, Cerveri P, Confalonieri N. An uncommon cause of cemented unicompartmental knee arthroplasty failure: fracture of metallic components. Knee Surg Sports Traumatol Arthrosc. 2013;21(11):2518-22.

30. Chan WCMP, Cooper AS, Glasgow MM, Donell ST, Walton NP. One-stage versus two-stage bilateral unicompartmental knee replacement: a comparison of immediate post-operative complications. J Bone Joint Surg Br. 2009:91(10):1305-9.

31. Pulido LGE, Joshi A, Purtill JJ, Parvizi J. Periprosthetic joint infection: the incidence, timing, and predisposing factors. Clin Orthop Relat Res. 2008:466(7):1710-5.

32. Del Pozo JLPR. Clinical practice. Infection associated with prosthetic joints. N Engl J Med. 2009;361(8):784-94.

33. Morris MJ, Molli RG, Berend KR, Lombardi AV Jr. Mortality and perioperative complications after unicompartmental knee arthroplasty. Knee. 2013;20(3):218-20.

34. Gill GSMD, Joshi AB. Mortality following primary total knee arthroplasty. J Bone Joint Surg Am. 2003;85-A(3):432-5.

\section{Publisher's Note}

Springer Nature remains neutral with regard to jurisdictional claims in published maps and institutional affiliations. 